\title{
Accelerating Universe and Dynamical Compactification of Extra Dimensions
}

\author{
F. Darabi* \\ Department of Physics, Azarbaijan University of Tarbiat Moallem, 53714-161, Tabriz, Iran . \\ Institute for Theoretical and Mathematical Physics, Niavaran, 19395-5746, Tehran, Iran.
}

\begin{abstract}
We study a $(4+D)$-dimensional Kaluza-Klein cosmology with a Robertson-Walker type metric having two scale factors $a$ and $R$, corresponding to $D$-dimensional internal space and 4-dimensional universe, respectively. By introducing an exotic matter as the space-time part of the higher dimensional energy-momentum tensor, a 4-dimensional decaying cosmological term is appeared as $\Lambda \sim R^{-2}$, playing the role of an evolving dark energy in the universe. The resulting field equations yield the exponential solutions for the scale factors. These exponential behaviors may account for the dynamical compactification of extra dimensions and the accelerating expansion of the 4-dimensional universe in terms of the Hubble parameter. The acceleration of universe may be explained by the negative pressure of the exotic matter. It is shown that the rate of compactification of higher dimensions depends on the dimension, $D$. We then obtain the Wheeler-DeWitt equation and find the general exact solutions in $D$-dimensions. A good correspondence between the classical solutions and the Wheeler-DeWitt solutions, in any dimension $D$, is obtained.
\end{abstract}

PACS number(s): 04.50.+h, 98.80.-k, 98.80.Qc

*e-mail: f.darabi@azaruniv.edu 


\section{Introduction}

The study of $\Lambda$-decaying cosmological models has recently been the subject of particular interest both from classical and quantum aspects. A dynamical characteristic for the cosmological term was already attributed by quantum field theorists since the developments in particle physics and inflationary scenarios. According to modern quantum field theory, the structure of a vacuum turns out to be interrelated with some spontaneous symmetry-breaking effects through the condensation of quantum (scalar) fields. This phenomenon gives rise to a nonvanishing vacuum energy density of the scale $M_{p}^{4}$ ( $M_{p}$ is the plank mass). Therefore, the bare cosmological constant may receive potential contributions from this mass scale resulting in a large effective cosmological constant which is too far from its present upper band. This is known as the cosmological constant problem. The $\Lambda$ decaying models may serve as potential candidates to solve this problem by decaying the large value of the cosmological constant $\Lambda$ to its present observed value.

Also, there are strong (astronomical) observational motivations for considering cosmological models in which $\Lambda$ is dynamically decreasing as $\Lambda \sim R^{-m}$. Some models assume a priori a fixed value for the parameter $m$. The case $m=2$, corresponding to the cosmic string matter, has mostly been taken based on dimensional considerations by some authors [1]. The case $m \approx 4$ which resembles the ordinary radiation has also been considered by some other authors [2]. A third group of authors have also studied the case $m=3$ corresponding to the ordinary matter [3]. There are also some other models in which the value of $m$ is not fixed a priori and the numerical bounds on the value of $m$ is estimated by observational data or obtained by calculation of the quantum tunnelling rate [4]. Other aspects of $\Lambda$-decaying models have also been discussed with no specific numerical bounds on $m$ [5].

On the other hand, the idea that our 4-dimensional universe might have emerged from a higher dimensional space-time is now receiving much attention [6] where the compactification of higher dimensions plays a key role. Meanwhile, the recent distance measurements of type Ia supernova suggest strongly an accelerating universe [7]. This accelerating expansion is generally believed to be driven by an energy source called dark energy which provides negative pressure, such as a positive cosmological constant [8], or a slowly evolving real scalar field called quintessence [9]. Moreover, the basic conclusion from all previous observations that $\sim$ 70 percent of the energy density of the universe is in a dark energy sector, has been confirmed after the recent WMAP [10].

To model a universe based on these considerations one may start from a fundamental theory including both gravity and standard model of particle physics. In this regards, it is interesting to begin with ten or eleven-dimensional space-time of superstring/M-theory, in which case one needs a compactification of ten or eleven-dimensional supergravity theory where an effective 4-dimensional cosmology undergoes acceleration. However, it has been known for some time that it is difficult to derive such a cosmology and has been considered that there is a no-go theorem that excludes such a possibility, if one takes the internal space to be time-independent and compact without boundary [11]. However, it has recently been shown that one may avoid this no-go theorem by giving up the condition of time-independence of the internal space; and a solution of the vacuum Einstein equations with compact hyperbolic internal space has been proposed based on this model[12]. Similar accelerating cosmologies can also be obtained for 
SM2 and SD2 branes, not only for hyperbolic but also for flat internal space[13].

On the other hand, from cosmological point of view, it is not so difficult to find cosmological models in which the 4-dimensional universe undergoes an accelerating expansion and the internal space contracts with time, exhibiting the dynamical compactification [14], [15], [16].

In [16], for instance, it is shown that using a more general metric, as compared to ref [14], and introducing matter without specifying its nature, the size of compact space evolves as an inverse power of the radius of the universe. The Friedmann-Robertson-Walker equations of the standard four-dimensional cosmology is obtained using an effective pressure expressed in terms of the components of the higher dimensional energy-momentum tensor, and the negative value of this pressure may explain the acceleration of our present universe.

To the author's knowledge the question of $\Lambda$-decaying cosmological model has not received much attention in higher dimensional Kaluza-Klein cosmologies. Moreover, the exotic matter has not been considered as an alternative candidate to produce the acceleration of the universe. The purpose of the present paper is to study a $(4+D)$-dimensional Kaluza-Klein cosmology ,with an extended Robertson-Walker type metric, in this context. As we are concerned with cosmological solutions, which are intrinsically time dependent, we may suppose that the internal space is also time dependent. It is shown that by taking this higher dimensional metric and introducing a 4-dimensional exotic matter, a decaying cosmological term $\Lambda \sim R^{-2}$ is appeared as a type of dark energy and the resulting field equations yield the exponential solutions for the scale factors of the four-dimensional universe and the internal space. These solutions may account for the accelerating universe and dynamical compactification of extra dimensions, driven by the negative pressure of the exotic matter ${ }^{1}$. It should be noted, however, that the solutions in principle describe typical inflation rather than the recently observed acceleration of the universe which is known to take place in an ordinary matter dominated universe. Nevertheless, regarding the fact that about 70 percent of the total energy density of the universe is of dark energy type with negative pressure, we may approximate the matter content of the universe with almost dark energy and consider the present model as a too simplified model of a real accelerating universe.

The quantum cosmology of this model is also studied by obtaining the Wheeler-DeWitt equation and finding its solutions. It is then shown that a good correspondence exists between the classical and quantum cosmological solutions.

The paper is organized as follows: In section $\mathbf{2}$, we introduce the model by taking a higher dimensional Robertson-Walker type metric and a higher dimensional matter whose non-zero part is a four-dimensional exotic matter. In section 3, we obtain the Einstein equations for the two scale factors. In section $\mathbf{4}$, we solve the Einstein equations and obtain the solutions. In section 5, we derive the Wheeler-DeWitt equation, and in section $\mathbf{6}$, the exact solutions of the Wheeler-DeWitt equation is obtained. Finally, in section 7, we show a good correspondence between the classical and quantum cosmology. The paper is ended with a conclusion.

\footnotetext{
${ }^{1} \mathrm{~A}$ similar work [17] has already been done in which the same extended FRW metric was chosen with a radiation fluid occupying all the extended space-time. They found an inflation for 3-dimensions and a contraction for the $D$ remaining spatial dimensions.
} 


\section{The model}

We start with the metric considered in [18], in which the space-time is assumed to be of Robertson-Walker type having an internal space with dimension $D$. We adopt a real chart $\left\{t, r^{i}, \rho^{a}\right\}$ with $t, r^{i}$, and $\rho^{a}$ denoting the time, space coordinates and internal space dimensions, respectively. We, therefore, take ${ }^{2}$

$$
d s^{2}=-N^{2}(t) d t^{2}+R^{2}(t) \frac{d r^{i} d r^{i}}{\left(1+\frac{k r^{2}}{4}\right)^{2}}+a^{2}(t) \frac{d \rho^{a} d \rho^{a}}{\left(1+k^{\prime} \rho^{2}\right)},
$$

where $N(t)$ is the lapse function, $R(t)$ and $a(t)$ are the scale factor of the universe and the radius of internal space, respectively; $r^{2} \equiv r^{i} r^{i}(i=1,2,3), \rho^{2} \equiv \rho^{a} \rho^{a}(a=1, \ldots D)$, and $k, k^{\prime}=0, \pm 1$. We assume the internal space to be flat with compact topology $S^{D}$, which means $k^{\prime}=0$. This assumption is motivated by the possibility of the compact spaces to be flat or hyperbolic in "accelerating cosmologies from compactification" scenarios, as discussed above.

The form of energy-momentum tensor is dictated by Einstein's equations and by the symmetries of the metric (1). Therefore, we may assume

$$
T_{A B}=\operatorname{diag}\left(-\rho, p, p, p, p_{D}, p_{D}, \ldots, p_{D}\right),
$$

where $A$ and $B$ run over both the space-time coordinates and the internal space dimensions. Now, we examine the case for which the pressure along all the extra dimensions vanishes, $p_{D}=0$. In so doing, we are motivated by the brane world scenarios where the matter is to be confined to the 4-dimensional universe, so that all components of $T_{A B}$ is set to zero but the space-time components [19] and it means no matter escapes through the extra dimensions.

We assume the energy-momentum tensor $T_{\mu \nu}$ of space-time to be an exotic $\chi$ fluid with the equation of state

$$
p_{\chi}=\left(\frac{m}{3}-1\right) \rho_{\chi},
$$

where $p_{\chi}$ and $\rho_{\chi}$ are the pressure and density of the fluid, respectively and the parameter $m$ is restricted to the range $0 \leq m \leq 2$ [20]. It is worth noting that the equation of state (3) with $0 \leq m \leq 2$ resembles a universe with negative pressure matter, violating the strong energy condition [21] and this violation is required for a universe to be accelerated [12].

Using standard techniques we obtain the scalar curvature corresponding to the metric (1) and then substitute the result into the dimensionally extended Einstein-Hilbert action ( without higher dimensional cosmological term) plus a matter term indicating the above mentioned exotic fluid. This leads to the effective Lagrangian ${ }^{3}$

$$
L=\frac{1}{2 N} R a^{D} \dot{R}^{2}+\frac{D(D-1)}{12 N} R^{3} a^{D-2} \dot{a}^{2}+\frac{D}{2 N} R^{2} a^{D-1} \dot{R} \dot{a}-\frac{1}{2} k N R a^{D}+\frac{1}{6} N \rho_{\chi} R^{3} a^{D},
$$

\footnotetext{
${ }^{2}$ There is a little difference between this metric and that of [18], in that here the lapse function is generally considered as $N(t)$ instead of taking $N=1$.

${ }^{3}$ We take the Planck units, $G=c=\hbar=1$
} 
where a dot represents differentiation w.r.t $t$. We now take a closed $(k=1)$ universe. It is easily shown that substituting the equation of state (3) into the continuity equation ${ }^{4}$

$$
\dot{\rho}_{\chi} R+3\left(p_{\chi}+\rho_{\chi}\right) \dot{R}=0
$$

leads to the following behavior of the energy density in a closed $(k=1)$ Friedmann-RobertsonWalker universe [20]

$$
\rho_{\chi}(R)=\rho_{\chi}\left(R_{0}\right)\left(\frac{R_{0}}{R}\right)^{m}
$$

where $R_{0}$ is the value of the scale factor at an arbitrary reference time $t_{0}$.

Now, we may define the cosmological term [4]

$$
\Lambda \equiv \rho_{\chi}(R)
$$

which leads to

$$
L=\frac{1}{2 N} R a^{D} \dot{R}^{2}+\frac{D(D-1)}{12 N} R^{3} a^{D-2} \dot{a}^{2}+\frac{D}{2 N} R^{2} a^{D-1} \dot{R} \dot{a}-\frac{1}{2} N R a^{D}+\frac{1}{6} N \Lambda R^{3} a^{D},
$$

where the cosmological term is now decaying with the scale factor $R$ as

$$
\Lambda(R)=\Lambda\left(R_{0}\right)\left(\frac{R_{0}}{R}\right)^{m}
$$

Note that $\Lambda$ is now playing the role of an evolving dark energy [22] in 4-dimensions, because we did not consider explicitly a $(4+D)$ dimensional cosmological term in the action, and $\Lambda$ appears merely due to the specific choice of the equation of state (3) for the exotic matter. The decaying $\Lambda$ term may also explain the smallness of the present value of the cosmological constant since as the universe evolves from its small to large sizes the large initial value of $\Lambda$ decays to small values.

Of particular interest, to the present paper, among the different values of $m$ is $m=2$ which has some interesting implications in reconciling observations with inflationary models [23], and is consistent with quantum tunnelling [4].

\section{$3 \quad$ Einstein equations}

We take $m=2$ and set the initial values of $R_{0}$ and $\Lambda\left(R_{0}\right)$

$$
\Lambda\left(R_{0}\right) R_{0}^{2}=3 \quad, \quad \Lambda(R)=\frac{3}{R^{2}}
$$

leading to a positive cosmological term which, according to (7), guarantees the weak energy condition $\rho_{\chi}>0$.

\footnotetext{
${ }^{4}$ To obtain this equation we may use the contracted Bianchi identity in $(4+\mathrm{D})$ dimensions, namely $\nabla_{M} G^{M N}=\nabla_{M} T^{M N}=0$ together with $T_{a b}=T_{\mu a}=0$ which gives rise to $\nabla_{\mu} T^{\mu \nu}=0$.
} 
The lapse function $N(t)$, in principle, is also an arbitrary function of time due to the reparametrization invariance of the action. We, therefore, take the gauge

$$
N(t)=R^{3}(t) a^{D}(t)
$$

Now, the Lagrangian becomes

$$
L=\frac{1}{2} \frac{\dot{R}^{2}}{R^{2}}+\frac{D(D-1)}{12} \frac{\dot{a}^{2}}{a^{2}}+\frac{D}{2} \frac{\dot{R} \dot{a}}{R a},
$$

where Eq.(10) has been used. It is seen that the parameters $k$ and $\Lambda$ are effectively removed from the Lagrangian and this implies that although $k$ and $\Lambda$ are not zero in this model the corresponding 4-dimensional universe is equivalent to a flat universe with a zero cosmological term. In other words, we do not distinguish between our familiar 4-dimensional universe, which seems to be flat and without any exotic fluid, and a closed universe filled with an exotic fluid. We now define the new variables

$$
X=\ln R, \quad Y=\ln a .
$$

The lagrangian (12) is written as

$$
L=\frac{1}{2} \dot{X}^{2}+\frac{D(D-1)}{12} \dot{Y}^{2}+\frac{D}{2} \dot{X} \dot{Y}
$$

The equations of motion are obtained

$$
\begin{gathered}
\ddot{X}+\frac{D}{2} \ddot{Y}=0, \\
\ddot{X}+\frac{D-1}{3} \ddot{Y}=0 .
\end{gathered}
$$

Combining the equations (15) and (16) we obtain

$$
\begin{aligned}
& \ddot{X}=0, \\
& \ddot{Y}=0 .
\end{aligned}
$$

\section{Solutions}

The solutions for $R(t)$ and $a(t)$ are as follows

$$
\begin{aligned}
& R(t)=A e^{\alpha t}, \\
& a(t)=B e^{\beta t},
\end{aligned}
$$

where the constants $A, B, \alpha$ and $\beta$ should be obtained, in principle, in terms of the initial conditions. It is a reasonable assumption that the size of all spatial dimensions be the same at $t=0$. Moreover, it may be assumed that this size would be the Planck size $l_{p}$ in accordance 
with quantum cosmological considerations. Therefore, we take $R(0)=a(0)=l_{P}$ so that $A=B=l_{p}$, and

$$
\begin{aligned}
& R(t)=l_{p} e^{\alpha t}, \\
& a(t)=l_{p} e^{\beta t} .
\end{aligned}
$$

It is important to note that the constants $\alpha, \beta$ are not independent, and a relation may be obtained between them. This is done by imposing the zero energy condition $H=0$ which is the well-known result in cosmology as a time reparametrization invariant theory. The Hamiltonian constraint is obtained through the Legender transformation of the Lagrangian (14)

$$
H=\frac{1}{2} \dot{X}^{2}+\frac{D(D-1)}{12} \dot{Y}^{2}+\frac{D}{2} \dot{X} \dot{Y}=0
$$

which is written in terms of $\alpha$ and $\beta$ as

$$
H=\frac{1}{2} \alpha^{2}+\frac{D(D-1)}{12} \beta^{2}+\frac{D}{2} \alpha \beta=0 .
$$

This constraint is satisfied only for $\alpha \leq 0, \beta \geq 0$ or $\alpha \geq 0, \beta \leq 0$.

For $D \neq 1$, the case $\alpha=0$ or $\beta=0$ gives rise to time independent scale factors, namely $R=a=l_{P}$, which is not physically viable. We, therefore, choose $\alpha>0, \beta<0$ so that the universe and the internal space would expand and contract, respectively, in accordance with the present observations.

For the case $D=1$, we find

$$
\left\{\begin{array}{l}
\beta=\text { arbitrary } \\
\alpha=0
\end{array} \quad \text { or } \quad \alpha=-\beta .\right.
$$

The former is not physically viable, since it predicts no time evolution for the universe. The latter, however, may predict exponential expansion for $R(t)$, and exponential contraction for $a(t)$, both with the same exponent $\alpha>0$.

For the general case $D>1$, we find

$$
\alpha_{ \pm}=\frac{D \beta}{2}\left[-1 \pm \sqrt{1-\frac{2}{3}\left(1-\frac{1}{D}\right)}\right]
$$

which gives two positive values for $\alpha$ indicating two possible expanding universes provided $\beta<0$ which indicates the compactification of extra dimensions. Moreover, the values of $\alpha_{ \pm}$, for a given negative value of $\beta$, become larger for higher dimensions. Therefore, the universe expands more rapidly in both possibilities. On the contrary, for a given positive value of $\alpha$, indicating an expanding universe, the parameter $\beta$ may take two negative values

$$
\beta_{ \pm}=\frac{2 \alpha}{D}\left[-1 \pm \sqrt{1-\frac{2}{3}\left(1-\frac{1}{D}\right)}\right]^{-1}
$$

indicating two ways of compactification. Moreover, they become smaller for higher dimensions, exhibiting lower rates of compactification. 
To find the constants $\alpha, \beta$ we first obtain the Hubble parameter for $R(t)$

$$
H=\frac{\dot{R}}{R}=\alpha
$$

by which the constant $\alpha$ is fixed. The observed positive value of $H$ will then justify our previous assumption, $\alpha>0$. We may, therefore, write the solutions (21) and (22) in terms of the Hubble parameter $H$ as

$$
\begin{aligned}
& R(t)=l_{p} e^{H t}, \\
& a(t)=l_{p} e^{-H t},
\end{aligned}
$$

for $D=1$, and

$$
\begin{gathered}
R(t)=l_{p} e^{H t}, \\
a(t)_{ \pm}=l_{p} e^{\frac{2 H t}{D}\left[-1 \pm \sqrt{1-\frac{2}{3}\left(1-\frac{1}{D}\right)}\right]^{-1}},
\end{gathered}
$$

and

$$
\begin{gathered}
R_{ \pm}(t)=l_{p} e^{\frac{D \beta t}{2}\left[-1 \pm \sqrt{1-\frac{2}{3}\left(1-\frac{1}{D}\right)}\right]} \\
a(t)=l_{p} e^{\beta t}
\end{gathered}
$$

for $D>1$.

For a given $H>0$, it is seen that the solution corresponding to $D=1$ may predict an accelerating (de Sitter) universe and a contracting internal space with exactly the same rates. For $D>1$, in Eqs.(31) and (32), for a given $H>0$ in the exponent of $R(t)$ the exponent in $a(t)$ takes two negative values and becomes smaller for higher dimensions. This means that while the 4-dimensional (de Sitter) universe is expanding by the rate $H$, the higher dimensions may be compactified in two possible ways with different rates of compactification as a function of dimension, D. In Eqs.(33) and (34), on the other hand, for a given $\beta<0$ the exponent in $R(t)$ takes two positive values which become larger for higher dimensions. This also means that while the extra dimensions contract by the rate $\beta$, the universe may be expanded in two possible ways with different expansion rates as a function of $D$.

It is easy to show that the Lagrangian (14) ( or the equations of motion ) is invariant under the simultaneous transformation

$$
R \rightarrow R^{-1} \quad, \quad a \rightarrow a^{-1}
$$

which is consistent with the time reversal $t \rightarrow-t$. Therefore, four different phases of "expansioncontraction" for $R(t)$ and $a(t)$ are distinguished, Eqs.(31) - (34). One may prefer the "expanding $R(t)$ - contracting $a(t)$ " phase to "expanding $a(t)$ - contracting $R(t)$ " one, considering the present status of the $4 \mathrm{D}$ universe ${ }^{5}$.

\footnotetext{
${ }^{5}$ For the special case $D=3$, both the Lagrangian (14) and the Hamiltonian constraint (23) are invariant under the transformation

$$
a \rightarrow R \quad, \quad R \rightarrow a .
$$
}

Therefore, we have a dynamical symmetry between $R$ and $a$, namely

$$
a \leftrightarrow R .
$$


The deceleration parameter $q$ for the scale factor $R$ is obtained

$$
q=-\frac{\ddot{R} R}{\dot{R}^{2}}=-1
$$

Observational evidences not only do not rule out the negative deceleration parameter but also puts the limits on the present value of $q$ as $-1 \leq q<0$ [7]. Therefore, this negative value seems to favor a cosmic acceleration in the expansion of the universe.

In the expansion phase of the closed $(k=1)$ universe the cosmological term $\Lambda$ decays exponentially with time $t$ as

$$
\Lambda(t)=3 l_{p}^{-2} e^{-2 H t}
$$

whereas in the contraction phase $(t \rightarrow-t)$ it grows exponentially to large values so that at $t=0$ it becomes extremely large, of the order of $M_{p}^{2}$. This huge value of $\Lambda$ may be extinguished rapidly by assuming a sufficiently large Hubble parameter $H$, consistent with the present observations, to alleviate the cosmological constant problem.

\section{$5 \quad$ Wheeler-DeWitt equation}

To obtain the Wheeler-DeWitt equation we start with the Lagrangian (14). The conjugate momenta corresponding to $X$ and $Y$ are obtained

$$
\begin{gathered}
P_{X}=\frac{\partial L}{\partial \dot{X}}=\dot{X}+\frac{D}{2} \dot{Y}, \\
P_{Y}=\frac{\partial L}{\partial \dot{Y}}=\frac{D}{2} \dot{X}+\frac{D(D-1)}{6} \dot{Y},
\end{gathered}
$$

from which we obtain

$$
\begin{gathered}
\dot{X}=\frac{6}{D+2}\left[P_{X}\left(\frac{1-D}{3}\right)+P_{Y}\right], \\
\dot{Y}=\frac{6}{D(D-1)}\left[P_{Y} \frac{2(1-D)}{D+2}-P_{X} \frac{D(1-D)}{D+2}\right] .
\end{gathered}
$$

Substituting Eqs.(40), (41) into the Hamiltonian constraint (23), we obtain

$$
H=(1-D) P_{X}^{2}-\frac{6}{D} P_{Y}^{2}+6 P_{X} P_{Y}=0 .
$$

Now, we may use the following quantum mechanical replacements

$$
P_{X} \rightarrow-i \frac{\partial}{\partial X} \quad, \quad P_{Y} \rightarrow-i \frac{\partial}{\partial Y}
$$

In this case there is no real line of demarcation between $a$ and $R$ to single out one of them as the real scale factor of the universe. This is because the internal space is flat $k^{\prime}=0$ and according to (12) one may assume the $4 \mathrm{D}$ universe with $k, \Lambda \neq 0$ to be equivalent to the one in which $k=\Lambda=0$. Therefore, both have the same topology $S^{3}$. 
by which the Wheeler-DeWitt equation is obtained

$$
\left[(D-1) \frac{\partial^{2}}{\partial X^{2}}+\frac{6}{D} \frac{\partial^{2}}{\partial Y^{2}}-6 \frac{\partial}{\partial X} \frac{\partial}{\partial Y}\right] \Psi(X, Y)=0
$$

where $\Psi(X, Y)$ is the wave function of the universe in the $(X, Y)$ mini-superspace.

We introduce the following change of variables

$$
x=X\left(1-\frac{D}{D+3}\right)+\frac{D}{D+3} Y \quad, \quad y=\frac{X-Y}{D+3},
$$

by which the Wheeler-DeWitt equation takes a simple form

$$
\left\{-3 \frac{\partial^{2}}{\partial x^{2}}+\frac{D+2}{D} \frac{\partial^{2}}{\partial y^{2}}\right\} \Psi(x, y)=0 .
$$

Now, we can separate the variables as $\Psi(x, y)=\phi(x) \psi(y)$ to obtain the following equations

$$
\begin{gathered}
\frac{\partial^{2} \phi(x)}{\partial x^{2}}=\frac{\gamma}{3} \phi(x), \\
\frac{\partial^{2} \psi(y)}{\partial y^{2}}=\frac{\gamma D}{D+2} \psi(y),
\end{gathered}
$$

where we assume $\gamma>0$.

\section{Solutions}

The solutions of Eqs.(46), (47) in terms of $x, y$ are as follows

$$
\begin{gathered}
\phi(x)=e^{ \pm \sqrt{\frac{\gamma}{3}} x}, \\
\psi(y)=e^{ \pm \sqrt{\frac{\gamma D}{D+2}} y},
\end{gathered}
$$

leading to the four possible solutions for $\Psi(x, y)$ as

$$
\begin{aligned}
& \Psi_{D}^{ \pm}(x, y)=A^{ \pm} e^{ \pm \sqrt{\frac{\gamma}{3}} x \pm \sqrt{\frac{\gamma D}{D+2}} y}, \\
& \Psi_{D}^{ \pm}(x, y)=B^{ \pm} e^{ \pm \sqrt{\frac{\gamma}{3}} x \mp \sqrt{\frac{\gamma D}{D+2}} y}
\end{aligned}
$$

or alternative solutions in terms of $X, Y$ as

$$
\begin{aligned}
& \Psi_{D}^{ \pm}(X, Y)=A^{ \pm} e^{ \pm \sqrt{\frac{\gamma}{3}}\left(\frac{3 X+D Y}{D+3}\right) \pm \sqrt{\frac{\gamma D}{D+2}}\left(\frac{X-Y}{D+3}\right)}, \\
& \Psi_{D}^{ \pm}(X, Y)=B^{ \pm} e^{ \pm \sqrt{\frac{\gamma}{3}}\left(\frac{3 X+D Y}{D+3}\right) \mp \sqrt{\frac{\gamma D}{D+2}}\left(\frac{X-Y}{D+3}\right)},
\end{aligned}
$$


where $A^{ \pm}, B^{ \pm}$are the normalization constants. We may also write down the solutions in terms of $R$ and $a^{6}$

$$
\begin{aligned}
& \Psi_{D}^{ \pm}(R, a)=A^{ \pm} R^{ \pm \frac{1}{D+3}\left(\sqrt{3 \gamma}+\sqrt{\frac{\gamma D}{D+2}}\right) a^{ \pm \frac{1}{D+3}}\left(\sqrt{\frac{\gamma}{3}} D-\sqrt{\frac{\gamma D}{D+2}}\right)} \\
& \Psi_{D}^{ \pm}(R, a)=B^{ \pm} R^{ \pm \frac{1}{D+3}\left(\sqrt{3 \gamma}-\sqrt{\frac{\gamma D}{D+2}}\right) a^{ \pm \frac{1}{D+3}}\left(\sqrt{\frac{\gamma}{3}} D+\sqrt{\frac{\gamma D}{D+2}}\right)} .
\end{aligned}
$$

It is now important to impose the good boundary conditions on the above solutions to single out the physical ones. In so doing, we may impose the following condition

$$
\Psi_{D}(R \rightarrow \infty, a \rightarrow \infty)=0
$$

Then, one may take the following solutions

$$
\Psi_{D}^{ \pm}(R, a)=C^{ \pm} R^{-\frac{1}{D+3}\left(\sqrt{3 \gamma} \pm \sqrt{\frac{\gamma D}{D+2}}\right)} a^{-\frac{1}{D+3}\left(\sqrt{\frac{\gamma}{3}} D \mp \sqrt{\frac{\gamma D}{D+2}}\right)}
$$

where $C^{ \pm}$are the normalization constants and the exponents of $R$ and $a$ are negative for any value of $D^{7}$.

One may obtain the solutions $(57)$ in $(X, Y)$ mini-superspace as

$$
\Psi_{D}^{ \pm}(X, Y)=C^{ \pm} e^{-\sqrt{\frac{\gamma}{3}}\left(\frac{3 X+D Y}{D+3}\right) \mp \sqrt{\frac{\gamma D}{D+2}}\left(\frac{X-Y}{D+3}\right)} .
$$

\section{Classical limits}

One of the most interesting topics in the context of quantum cosmology is the mechanisms through which classical cosmology may emerge from quantum cosmology. When does a Wheeler-DeWitt wave function predict a classical space-time? Hartle has put forward a simple rule for applying quantum mechanics to a single system (universe): If the wave function is sufficiently peaked about some region in the configuration space we predict to observe a correlation between the observables which characterize this region.

In this regards, we first take $D=1$ and look for a correspondence between classical and quantum solutions. Using Eqs.(29) and (30) in the Planck units, the corresponding classical locus in $(R, a)$ configuration space, is

$$
R a=1
$$

whereas in $(X, Y)$ coordinates we have

$$
X+Y=0 \text {. }
$$

We now consider the wave functions $(58)$ in $(X, Y)$ mini-superspace for $D=1$

$$
\Psi_{1}^{+}(X, Y)=C^{+} e^{-\sqrt{\frac{\gamma}{3}} X}
$$

\footnotetext{
${ }^{6}$ For $D=3$, there is a exchange symmetry $\Psi(R, a) \leftrightarrow \Psi(a, R)$ under the exchange $a \leftrightarrow R$.

${ }^{7}$ For $D=1$, the exponent of " $a$ " corresponding to $\Psi^{+}$becomes zero so that $\Psi^{+}$depends only on $R$ with the condition $\Psi^{+}(R \rightarrow \infty) \rightarrow 0$.
} 


$$
\Psi_{1}^{-}(X, Y)=C^{-} e^{-\sqrt{\frac{\gamma}{3}} \frac{X+Y}{2}} .
$$

The above wave functions, in their present form, are not square integrable as is required for the wave functions to predict the classical limit. However, one may take the absolute value of the exponents to make the wave functions square integrable

$$
\begin{gathered}
\Psi_{1}^{+}(X, Y)=C^{+} e^{-\left|\sqrt{\frac{\gamma}{3}} X\right|}, \\
\Psi_{1}^{-}(X, Y)=C^{-} e^{-\left|\sqrt{\frac{\gamma}{3}} \frac{X+Y}{2}\right|} .
\end{gathered}
$$

We next consider the general case $D>1$. Eliminating the parameter $t$ in Eqs. (31) and (32) the classical loci in terms of $R, a$ are obtained

$$
a_{ \pm}=R^{\frac{2}{D}\left[-1 \pm \sqrt{1-\frac{2}{3}\left(1-\frac{1}{D}\right)}\right]^{-1}} .
$$

The corresponding forms of these loci in terms of $X, Y$ are

$$
\begin{aligned}
& Y_{+}=\frac{2}{D} X\left[-1+\sqrt{1-\frac{2}{3}\left(1-\frac{1}{D}\right)}\right]^{-1}, \\
& Y_{-}=\frac{2}{D} X\left[-1-\sqrt{1-\frac{2}{3}\left(1-\frac{1}{D}\right)}\right]^{-1} .
\end{aligned}
$$

The wave functions (58) also are not square integrable, so we may replace the exponents by their absolute values

$$
\Psi_{D}^{ \pm}(X, Y)=C^{ \pm} e^{-\mid \sqrt{\frac{\gamma}{3}\left(\frac{3 X+D Y}{D+3}\right) \mp \sqrt{\frac{\gamma D}{D+2}}\left(\frac{X-Y}{D+3}\right) \mid},}
$$

to make them square integrable. Now, following Hartle's point of view, we try to make correspondence between the classical loci and the wave functions.

Figures 1, 2, and 3 show respectively the 2 D plots of the typical wave functions $\Psi_{1}^{+}, \Psi_{3}^{+}$ and $\Psi_{9}^{+}$in terms of $(X, Y)$ for $\gamma=10^{-6}$; Figures 13, 14, and 15 show the corresponding 3D plots, respectively. On the other hand, Figures 7, 8, and 9 show the classical loci (60) for $D=1$, (66) for $D=3$ and $D=9$, respectively. It is seen that the $2 \mathrm{D}$ and $3 \mathrm{D}$ plots of the wave functions $\Psi_{1}^{+}, \Psi_{3}^{+}$and $\Psi_{9}^{+}$are exactly peaked on the classical loci.

In the same way, Figures 4, 5, and 6 show respectively the $2 \mathrm{D}$ plots of the wave functions $\Psi_{3}^{-}, \Psi_{6}^{-}$and $\Psi_{9}^{-}$. Figures 16,17 , and 18 show the corresponding 3D plots, respectively. Figures 10,11 , and 12 show the classical loci $(67)$ for $D=3, D=6$ and $D=9$, respectively. It is seen again an exact correspondence between the $2 \mathrm{D}$ and $3 \mathrm{D}$ plots of the wave functions $\Psi_{3}^{-}, \Psi_{6}^{-}$, $\Psi_{9}^{-}$and the classical loci. This procedure applies for all $D$. 


\section{Conclusion}

In this paper, we have studied a $(4+D)$-dimensional Kaluza-Klein cosmology with two scale factors, $R$ for the universe and $a$ for the higher dimensional space. By introducing a typical exotic matter in 4-dimensions, resulting in a decaying cosmological term, we obtain exponential solutions for $R$ and $a$ in terms of the Hubble parameter, indicating the accelerating expansion of the universe and dynamical compactification of extra dimensions, respectively. The rate of compactification is shown to be dependent on the number of extra dimensions, $D$. The more extra dimensions, the less rate of compactification. It is worth noting that the model is free of initial singularity problem because both $R$ and $a$ are non-zero at $t=0$, resulting in a finite Ricci scalar.

The model, although describes in principle a closed universe with non-vanishing cosmological constant, but is equivalent to a flat universe with zero cosmological constant. Therefore, one may assume that we are really living in a closed universe with $\Lambda \neq 0$, but it effectively appears as a flat universe with $\Lambda=0$. Note that we have not considered ordinary matter

sources in the model except an exotic matter source which is to be considered as a source of dark energy. Therefore, it seems the solutions to describe typical inflation rather than the recently observed acceleration of the universe which is known to take place in an ordinary matter dominated universe. However, if the large percent of the matter sources in the universe would be of dark energy type (as the present observations strongly recommend), then one may keep the results here even in the presence of other matter source, keeping in mind that the relevant contribution to the total matter source of the universe is the dark energy.

A question may arise on the fact that no physics is supposed to exist below the planck length whereas for the contracting solution, the scale factor $a(t)$ goes to zero starting from $l_{p}$. However, it is not a major problem because we have not considered elements of quantum gravity theory in this model and merely studied a model based on general relativity which is supposed to be valid in any scale without limitation. The scale $l_{p}$, in this paper, is not introduced within a quantum gravity model (action); it just appears as a typical initial condition, in the middle of a classical model, based on the quantum cosmological consideration. One may choose another scale based on some other physical considerations.

We have also studied the corresponding quantum cosmology and obtained the exact solutions. We have shown a good correspondence between the classical and quantum cosmological solutions for any $D$, provided that the wave functions vanish for the infinite scale factors. There is no such a correspondence if another boundary condition, other than stated, is taken.

\section{Acknowledgment}

The author would like to thank A. Rezaei-Aghdam and referees for useful and constructive comments. This work has been supported by the Research office of Azarbaijan University of Tarbiat Moallem, Tabriz, Iran. 


\section{References}

[1] J. L. Lopez, D. V. Nanopoulos, Mod. Phys. Lett. A11, 1 (1996); M. Özer, M. O. Taha, Phys. Lett. B171, 363 (1986); A.-M. M. Abdel-Rahman, Phys. Rev. D45, 3497 (1992); A. and R. G. Vishwakarma, Class. Quant. Grav. 14, 945 (1997); W. Chen, Y.-S. Wu, Phys. Rev. D41, 695 (1990); M. O. Calvao et al, Phys. Rev. D45, 3869 (1992); V. Méndez, D. Pavón, Gen. Rel. Grav. 28, 697 (1996).

[2] K. Freese et al, Nucl. Phys. B287, 797 (1987); M. Gasperini, Phys. Lett. B194, 347 (1987); J. M. Overduin, P. S. Wesson and S. Bowyer, Astrophys. J. 404, 1 (1993).

[3] F. Hoyle, G. Burbidge and J. V. Narlikar, Mon. Not. R. Astron. Soc. 286, 173 (1997).

[4] M. A. Jafarizadeh, F. Darabi, A. Rezaei-Aghdam and A. R. Rastegar, Phys. Rev. D60, 063514 (1999); T. S. Olson, T. F. Jordan, Phys. Rev. D35, 3258 (1987); V. Silveira, I. Waga, Phys. Rev. D50, 4890 (1994); Phys. Rev. D56, 4625 (1997); L. F. B. Torres, I. Waga, Mon. Not. R. Astron. Soc. 279, 712 (1996).

[5] M. D. Maia, G. S. Silva, Phys. Rev. D50, 7233 (1994); R. F. Sisteró, Gen. Rel. Grav. 23, 1265 (1991); J. Matyjasek, Phys. Rev. D51, 4154 (1995).

[6] T. Appelquist, A. Chodos and P. G. O. Freund, Modern Kaluza-Klein Theories, Frontiers in Physics Series, (Volume 65), 1986, (Ed. Addison-Wesely). A. G. Riess et al. Astrophys. J. 560, 49 (2001).

[7] S. Perlmutter et al., Bull. Am. Phys. Soc. 29, 1351 (1997); Ap. J. 507, 46 (1998); A. G. Riess et al., Astron. J. 116 (1998); P. M. Garnavich et al., Ap. J. Lett. 493, 53 (1998); Science 279, 1298 (1998); Ap. J. 509, 74 (1998); B. Schmidt et al., The High-Z Supernova Search: Measuring Cosmic Deceleration and Global Curvature of the Universe using Type IA Supernova, astro-ph/9805200.

[8] L. Krauss and M. S. Turner, Gen. Rel. Grav. 27, 1137 (1995); J. P. Ostriker and P. J. Steinhardt, Nature 377, 600 (1995); A. R. Liddle, D. H. Lyth, P. T. Viana and M. White, Mon. Not. Roy. Astron. Soc. 282, 281 (1996).

[9] R. R. Caldwell, R. Dave and P. J. Steinhardt, Phys. Rev. Lett. 80, 1582 (1998); M. C. Bento, O. Bertolami, Gen. Rel. Grav. 31, 1461 (1999); O. Bertolami, Nuovo Cimento, B93, 36 (1986).

[10] C. L. Benett et al., First Year Wilkinson Microwave Anisotropy Probe (WMAP) Observations: Preliminary Maps and Basic Results, astro-ph/0302207.

[11] G. W. Gibbons, Aspects of Supergravity Theories in Supersymmetry, Supergravity and Related Topics, (World Scientific Singapore, 1985); J. Maldacena, C. Nuñez, Int. J. Mod. Phys. A16, 822 (2001).

[12] P. K. Townsend, M. N. R. Wohlfarth, Accelerating Cosmologies from Compactification, hep-th/0303097. 
[13] L. Cornalba, M. Costa, Phys. Rev. D66, 066001 (2002); L. Cornalba, M. Costa, and C. Kounnas, Nucl. Phys. B637, 378 (2002); N. Ohta, hep-th/0303238; S. Roy, hepth/0304048.

[14] A. Chodos and S. Detweiler, Phys. Rev. D21, 2167 (1980); T. Dereli and R. W. Tucker, Phys. Lett. B125, 133 (1983); J. Gu, W. P. Hwang, Phys. Rev. D66, 024003 (2002).

[15] A. S. Majumdar and S. K. Sethi, Phys. Rev. D46, 5315 (1992); A. S. Majumdar, T. R. Seshadri and S. K. Sethi, Phys. Lett. B312, 67 (1993); A. S. Majumdar, Phys. Rev. D55, 6092 (1997); A. S. Majumdar, Ind. Jour. Phys.73 B, 843 (1999); A. S. Majumdar, Phys. Rev. D64, 083503 (2001).

[16] N. Mohammedi, Phys. Rev. D65, 104018 (2002).

[17] D. Sahdev, Phys. Lett. B137 155 (1984).

[18] J. Wudka, Phys. Rev. D35, 3255 (1987); Phys. Rev. D36, 1036 (1987).

[19] G. Dvali, G. Gabadadze, M. Porrati, Phys. Lett. B485, 208 (2000); B. Abdesselam, N. Mohemmedi, Phys. Rev. D665, 084018 (2002); N. Kaloper, J. March-Russell, G. D. Starkman and M. Trodden, Phys. Rev. Lett. 85, 928 (2000).

[20] D. Atkatz and H. Pagels, Phys. Rev. D25, 2065 (1982); Am. J. Phys. 62 (7), 619 (1994).

[21] M. A. Jafarizadeh, F. Darabi, A. Rezaei-Aghdam and A. R. Rastegar, Mod.Phys.Lett. A13, 3213 (1998).

[22] P. J. E. Peebles, B. Ratra, The cosmological constant and dark energy, astro-ph/0207347.

[23] W. Chen and Y. Wu, Phys. Rev. D41, 695 (1990). 


\section{Figure captions}

FIG. 1. 2D plot of $\Psi_{1}^{+}$in terms of $(X, Y)$ for $\gamma=10^{-6}$

FIG. 2. 2D plot of $\Psi_{3}^{+}$in terms of $(X, Y)$ for $\gamma=10^{-6}$

FIG. 3. 2D plot of $\Psi_{9}^{+}$in terms of $(X, Y)$ for $\gamma=10^{-6}$

FIG. 4. 2D plot of $\Psi_{3}^{-}$in terms of $(X, Y)$ for $\gamma=10^{-6}$

FIG. 5. 2D plot of $\Psi_{6}^{-}$in terms of $(X, Y)$ for $\gamma=10^{-6}$

FIG. 6. 2D plot of $\Psi_{9}^{-}$in terms of $(X, Y)$ for $\gamma=10^{-6}$

FIG. 7. Classical locus $X+Y=0$ for $D=1$

FIG. 8. Classical locus $Y_{+}=\frac{2}{D} X\left[-1+\sqrt{1-\frac{2}{3}\left(1-\frac{1}{D}\right)}\right]^{-1}$ for $D=3$

FIG. 9. Classical locus $Y_{+}=\frac{2}{D} X\left[-1+\sqrt{1-\frac{2}{3}\left(1-\frac{1}{D}\right)}\right]^{-1}$ for $D=9$

FIG. 10. Classical locus $Y_{-}=\frac{2}{D} X\left[-1-\sqrt{1-\frac{2}{3}\left(1-\frac{1}{D}\right)}\right]^{-1}$ for $D=3$

FIG. 11. Classical locus $Y_{-}=\frac{2}{D} X\left[-1-\sqrt{1-\frac{2}{3}\left(1-\frac{1}{D}\right)}\right]^{-1}$ for $D=6$

FIG. 12. Classical locus $Y_{-}=\frac{2}{D} X\left[-1-\sqrt{1-\frac{2}{3}\left(1-\frac{1}{D}\right)}\right]^{-1}$ for $D=9$

FIG. 13. 3D plot of $\Psi_{1}^{+}$in terms of $(X, Y)$ for $\gamma=10^{-6}$

FIG. 14. 3D plot of $\Psi_{3}^{+}$in terms of $(X, Y)$ for $\gamma=10^{-6}$

FIG. 15. 3D plot of $\Psi_{9}^{+}$in terms of $(X, Y)$ for $\gamma=10^{-6}$

FIG. 16. 3D plot of $\Psi_{3}^{-}$in terms of $(X, Y)$ for $\gamma=10^{-6}$

FIG. 17. 3D plot of $\Psi_{6}^{-}$in terms of $(X, Y)$ for $\gamma=10^{-6}$

FIG. 18. 3D plot of $\Psi_{9}^{-}$in terms of $(X, Y)$ for $\gamma=10^{-6}$ 
This figure "1.JPG" is available in "JPG" format from: http://arxiv.org/ps/gr-qc/0301075v2 
This figure "10.jpg" is available in "jpg" format from: http://arxiv.org/ps/gr-qc/0301075v2 
This figure "11.jpg" is available in "jpg" format from: http://arxiv.org/ps/gr-qc/0301075v2 
This figure "12.jpg" is available in "jpg" format from: http://arxiv.org/ps/gr-qc/0301075v2 
This figure "13.JPG" is available in "JPG" format from: http://arxiv.org/ps/gr-qc/0301075v2 
This figure "14.jpg" is available in "jpg" format from: http://arxiv.org/ps/gr-qc/0301075v2 
This figure "15.jpg" is available in "jpg" format from: http://arxiv.org/ps/gr-qc/0301075v2 
This figure "16.jpg" is available in "jpg" format from: http://arxiv.org/ps/gr-qc/0301075v2 
This figure "17.jpg" is available in "jpg" format from: http://arxiv.org/ps/gr-qc/0301075v2 
This figure "18.jpg" is available in "jpg" format from: http://arxiv.org/ps/gr-qc/0301075v2 
This figure "2.JPG" is available in "JPG" format from: http://arxiv.org/ps/gr-qc/0301075v2 
This figure "3.JPG" is available in "JPG" format from: http://arxiv.org/ps/gr-qc/0301075v2 
This figure "4.jpg" is available in "jpg" format from: http://arxiv.org/ps/gr-qc/0301075v2 
This figure "5.jpg" is available in "jpg" format from: http://arxiv.org/ps/gr-qc/0301075v2 
This figure "6.jpg" is available in "jpg" format from: http://arxiv.org/ps/gr-qc/0301075v2 
This figure "7.jpg" is available in "jpg" format from: http://arxiv.org/ps/gr-qc/0301075v2 
This figure "8.jpg" is available in "jpg" format from: http://arxiv.org/ps/gr-qc/0301075v2 
This figure "9.jpg" is available in "jpg" format from: http://arxiv.org/ps/gr-qc/0301075v2 